Document downloaded from:

http://hdl.handle.net/10251/63772

This paper must be cited as:

Casabán Bartual, MC.; Cortés López, JC.; Jódar Sánchez, LA. (2015). A random Laplace transform method for solving random mixed parabolic differential problems. Applied Mathematics and Computation. 259:654-667. doi:10.1016/j.amc.2015.02.091.

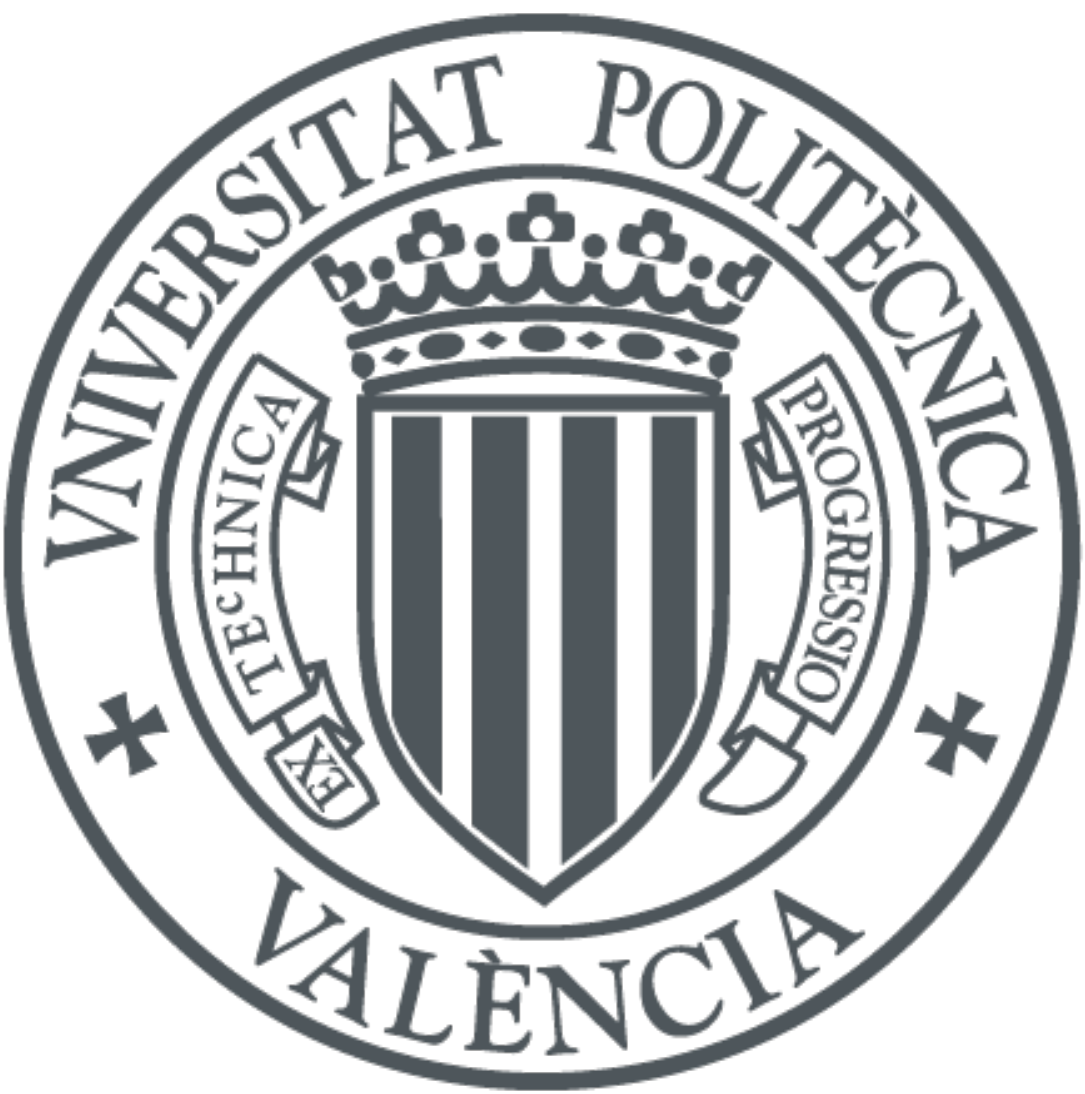

The final publication is available at

https://dx.doi.org/10.1016/j.amc.2015.02.091

Copyright Elsevier

Additional Information 


\title{
A random Laplace transform method for solving random mixed parabolic differential problems
}

\author{
M.-C. CasabÁn*, J.-C. Cortés, L. Jódar \\ Instituto Universitario de Matemática Multidisciplinar \\ Universitat Politècnica de València \\ Camino de Vera s/n, 46022 Valencia, Spain \\ jccortes@imm.upv.es (J.-C. Cortés); ljodar@imm.upv.es (L. Jódar)
}

\begin{abstract}
This paper deals with the explicit solution of random mixed parabolic equations in unbounded domains by using the random Laplace transform to second order stochastic processes. The mean square random Laplace operational calculus is stated and its application to the random parabolic equation together with previous results of the underlying random ordinary differential equations allow us to obtain an explicit solution of the problem. A numerical example, which includes simulations, illustrates the developed method.

Keywords: Random mixed parabolic equations, Random Laplace transform, Mean square and mean fourth random calculus.
\end{abstract}

\section{Introduction}

2 The integral transform method has proven its relevance to solve initial-boundary value problems for linear differential and integral equations. The essence of this success is based on its powerful operational calculus [1]-[9]. The required integral transform is closely related to the structure of the equation and the initial-boundary conditions of the problem. It is known that deterministic models are often a simplification of real problems to make more approachable their mathematical treatment. However, uncertainty is being incorporated into the mathematical modelling in different ways and points of view. For instance, spatial variability of geologic media properties involves geostatistical randomness and it has relevance in the analysis of fluid flows and solute transport, see [10]-[12]. In water resources problems there appear also random heterogeneous domains in the search of the solution process, see [13]-[15]. In this paper, we assume known uncertainty in the sense that some input parameters are assumed to be random variables (r.v.'s) and stochastic processes (s.p.'s) instead

*Corresponding author. Phone: +34 963879144. E-mail address: macabar@imm.upv.es 


\section{2 Preliminaries about $L_{p}$-calculus} random trigonometric Fourier transform, see [23, 24]. s.p. Specifically, this paper deals with the random heat problem

$$
\begin{aligned}
u_{t}(x, t) & =L u_{x x}(x, t), & t & >0, \quad x>0, \\
u(x, 0) & =0, & x & >0, \\
u(0, t) & =f(t ; A), & t & >0, \\
u(x, t) & \text { is bounded as } & x \rightarrow+\infty, & t>0,
\end{aligned}
$$
tions have a positive lower bound $\ell_{1}>0$, i.e,

$$
L(\omega) \geq \ell_{1}>0, \quad \forall \omega \in \Omega,
$$

of numbers and classical functions, respectively. Apart from modelling, there are several operational approaches to deal with continuous time uncertainty problems, namely, stochastic differential equations whose solution requires Itô or Stratonovich calculus [16]-[18] and, random differential equations for which the mean square calculus constitutes an adequate framework to conduct their analysis [19]. Stochastic advection-dispersion problems subject to random initial and boundary conditions have been studied in [20]-[22] using the moment method in the solution of nonreactive solute transport problems. Recently, the Fourier transform method has been applied to solve random partial differential problems, by introducing the random exponential Fourier transform and the

Stochastic Laplace transform extensions related to the Brownian motion and the Itô calculus throughout stochastic differential equations have been treated in [25] and more recently in [26]. In this paper, we extend to the random framework, the random Laplace transform and its random operational calculus to solve random partial differential models. As in the case of the random Fourier transforms [23, 24], we obtain an explicit mean square solution s.p. of the problem, as well as the expectation and the variance of the solution s.p. Apart from the mean square approach, other different approach based on the random variable transformation method has been used in [27] to deal with the transport equation and the computation of the probability density function of the solution

Throughout this paper, $(\Omega, \mathcal{F}, \mathcal{P})$ will denote a common probabilistic space where all r.v.'s and s.p.'s that appear in the problem under study are defined.

where $L$ is assumed to be a positive r.v., independent of r.v. $A$, whose realiza-

and $f(t ; A)$ is a s.p. which depends on one single r.v. $A$. The same results are available, but involving more complicated notation, by considering $f(t ; \cdot)$ a s.p. with a finite degree of randomness (see [19, p. 37] for comments in this regard).

For the sake of clarity, in this section we summarize some important concepts and results related to the so-called $L_{p}$-calculus, mainly focusing on the mean square (m.s.) and the mean fourth (m.f.) calculus, which correspond to $p=2$ 
and $p=4$, respectively (see $[19,28]$ for further details). Throughout this paper we will consider the set $L_{p}$, with $p \geq 1$, of all real-valued r.v.'s, $X$, defined on a probabilistic space $(\Omega, \mathcal{F}, \mathcal{P})$ such that $\mathrm{E}\left[|X|^{p}\right]<+\infty$, where $\mathrm{E}[\cdot]$ denotes the expectation operator. For short, in the sequel these r.v.'s will be referred to as $p$-r.v.'s. It can be proven that the space $L_{p}$ endowed with the following norm $\|X\|_{p}=\left(\mathrm{E}\left[|X|^{p}\right]\right)^{1 / p}$ is a Banach space, [29, p.9]. Throughout this paper $\|\cdot\|_{p}$ will be termed $p$-norm.

The definition of $p$-convergence of a sequence $\left\{X_{n}: n \geq 0\right\}$ of $p$-r.v.'s to the r.v. $X \in L_{p}$, is the one inferred by the $p$-norm, i.e., $\lim _{n \rightarrow+\infty}\left\|X_{n}-X\right\|_{p}=0$. The particular cases $p=2$ and $p=4$ are referred to as mean square (m.s.) and mean fourth (m.f.) convergence, respectively, and they are ones to be used throughout this paper.

It can be proven the following key inequality (see [30])

$$
\|X Y\|_{2} \leq\|X\|_{4}\|Y\|_{4}, \quad X, Y \in L_{4}
$$

which permits to establish that m.f. convergence entails m.s. convergence by specializing it for $Y=1$. Note that it also proves that $L_{4} \subseteq L_{2}$. The role of functions in the space $L_{p}$ are played by stochastic processes, which are defined by a family of $p$-r.v.'s indexed by a set of indexes $t \in T \subset \mathbb{R}$, i.e., a family $\{X(t): t \in T\}$ of real r.v.'s such as $\mathrm{E}\left[|X(t)|^{p}\right]<+\infty, \forall t \in T$ is called a $p$-stochastic process. The definitions of $p$-th mean continuity, $p$-th mean differentiability and $p$-th mean integrability follow straightforwardly from the ones inferred by the $p$-norm. For instance, in accordance with [19, p. 99], [31], we say that a s.p. $\{X(t): t \in \mathbb{R}\}$ with $X(t) \in L_{p}$ for all $t$, is $L_{p}$-locally integrable in $\mathbb{R}$ if, for all finite interval $\left[t_{1}, t_{2}\right] \subset \mathbb{R}$, the integral $\int_{t_{1}}^{t_{2}} X(t) d t$ exits in $L_{p}$.

In dealing with random differential equations, it is exceptional to obtain closed solutions but reliable approximations from which the main statistical properties, such the mean and variance, are computed. The mean square convergence has the following desirable property regarding the computation of reliable approximations to the exact mean and variance (see Theorems 4.2.1 and 4.3.1. in [19]).

Lemma 1 Let $\left\{X_{n}: n \geq 0\right\}$ and $\left\{Y_{m}: m \geq 0\right\}$ be two sequences of 2-r.v.'s m.s. convergent to $X \in L_{2}$ and $Y \in L_{2}$, respectively, i.e.,

$$
\lim _{n \rightarrow \infty}\left\|X_{n}-X\right\|_{2}=0, \quad \lim _{m \rightarrow \infty}\left\|Y_{m}-Y\right\|_{2}=0 .
$$

Then,

$$
\lim _{n, m \rightarrow \infty} \mathrm{E}\left[X_{n} Y_{m}\right]=\mathrm{E}[X Y]
$$

In particular,

$$
\lim _{n \rightarrow \infty} \mathrm{E}\left[X_{n}\right]=\mathrm{E}[X], \quad \lim _{n \rightarrow \infty} \operatorname{Var}\left[X_{n}\right]=\operatorname{Var}[X] .
$$


The following result is a straightforward consequence of inequality (6) that will be required later.

Lemma 2 Let $D$ be a 4-r.v. and, let $g(t)$ be a 4-s.p. verifying that $\lim _{t \rightarrow \infty}\|g(t)\|_{4}=0$. Then

$$
\lim _{t \rightarrow \infty}\|D g(t)\|_{2}=0 .
$$

We recall that the absolute moment of a real-valued r.v. $X$ coincides with the absolute moment of r.v. $i X$, where $i=\sqrt{-1}$ denotes the imaginary unit, i.e.,

$$
\mathrm{E}\left[|i X|^{n}\right]=\mathrm{E}\left[|X|^{n}\right], \quad n \geq 0 .
$$

As usual, $\operatorname{Re}(s)$ and $\operatorname{Im}(s)$ will denote the real and imaginary parts, respectively, of a complex number $s=x+i y, x, y \in \mathbb{R}$.

Finally, we remember that if $X$ is an absolute r.v. defined on the domain $\mathcal{D}(X)$ whose p.d.f. is $g_{X}(x)$, and one considers a transformed r.v. by the mapping $h$, say $Y=h(X)$, then the expectation of r.v. $Y$ can be computed as follows

$$
\mathrm{E}[Y]=\int_{\mathcal{D}(X)} h(x) g_{X}(x) d x .
$$

\section{Random Laplace transform and its operational calculus}

In this section, we introduce the random Laplace transform of a 2-s.p. and we show some s.p.'s which admit Laplace transform including the computation of its value. Finally, we give some operational rules to the random Laplace transform that will be required in the next section to solve the random heat problem (1)-(4).

Definition 1 Let us introduce the class $\mathfrak{C}$ of all the 2-s.p.'s $f(t)$ defined in the real line such that:

(i) $f(t)$ is m.s. locally integrable,

(ii) $f(t)=0$, if $t<0$,

(iii) The 2-norm of $f(t)$ is of exponential order, i.e., there exist constants $a \geq 0$ and $M>0$ such that

$$
\|f(t)\|_{2} \leq M e^{a t}, \quad t \geq 0 .
$$

Then, the random Laplace transform of a 2-s.p. $f(t) \in \mathfrak{C}$ is defined by the m.s. integral

$$
F(s)=\mathcal{L}[f(t)](s)=\int_{0}^{\infty} f(t) e^{-s t} d t, \quad s \in \mathbb{C}, \quad \operatorname{Re}(s)>a \geq 0 .
$$


Note that the integral (14) is well-defined in the half-plane $\operatorname{Re}(s)>a$ because from (13) one gets

$$
\left\|f(t) e^{-s t}\right\|_{2}=\|f(t)\|_{2} e^{-\operatorname{Re}(s) t} \leq M e^{(a-\operatorname{Re}(s)) t},
$$

and, consequently

$$
\int_{0}^{\infty}\left\|f(t) e^{-s t}\right\|_{2} d t \leq M \int_{0}^{\infty} e^{(a-\operatorname{Re}(s)) t} d t<+\infty .
$$

For the sake of convenience, let us recall that the Heaviside function $H(t)$ is defined as

$$
H(t)= \begin{cases}0, & t<0 \\ 1, & t \geq 0\end{cases}
$$

If $f(t)$ is a 2-s.p. in the class $\mathfrak{C}$, then $f(t) H(t)$ is in $\mathfrak{C}$, too.

Next, we provide several examples with the aim to show that the random Laplace transform can be applied to a wide range of s.p.'s under certain conditions that will be determined later. Example 1 involves an exponential s.p., Example 2 deals with a trigonometric s.p. and, finally Example 3 contains a s.p. that will be play an important role in the resolution of problem (1)-(4).

Example 1 Let $B$ be a real-valued r.v. satisfying that

$$
\exists \alpha>0: \quad \mathrm{E}\left[|B|^{n}\right]=\mathcal{O}\left(\alpha^{n}\right), \quad \forall n \geq 0,
$$

then, we shall show that the s.p.

$$
v_{1}(t ; B)=e^{B t} H(t),
$$

where $H(t)$ is the Heaviside function defined by (15), admits a random Laplace transform for $\operatorname{Re}(s)>\alpha$.

In fact, by (16) there exists $c>0$, such that

$$
\begin{aligned}
\left(\left\|e^{B t}\right\|_{4}\right)^{4} & =\mathrm{E}\left[e^{4 B t}\right]=\mathrm{E}\left[\sum_{n \geq 0} \frac{(4 B t)^{n}}{n !}\right] \leq \sum_{n \geq 0} \frac{4^{n} t^{n}}{n !} \mathrm{E}\left[|B|^{n}\right] \\
& \leq c \sum_{n \geq 0} \frac{(4 t \alpha)^{n}}{n !}=c e^{4 \alpha t} .
\end{aligned}
$$

Then, using (6) one gets,

$$
\left\|e^{B t}\right\|_{2} \leq\left\|e^{B t}\right\|_{4} \leq \sqrt[4]{c} e^{\alpha t} .
$$

Since the infinite series in (18) is m.f. convergent, and hence, m.s. convergent, the application of property (9) guarantees the commutation between the expectation operator and the infinite series in (18). 
Thus, the s.p. $v_{1}(t ; B)$ satisfies properties (i)-(iii) of Definition 1 with $M=\sqrt[4]{c}>0$ and $a=\alpha>0$, and its random Laplace transform, denoted by $\mathcal{L}\left[v_{1}(t ; B)\right](s)$, exists for $\operatorname{Re}(s)>\alpha$. Now, in order to compute it we first consider $s \in \mathbb{R}$ such that $s>\alpha$ and then applying the fundamental theorem of m.s. calculus, [19, p. 104], one gets

$$
\mathcal{L}\left[v_{1}(t ; B)\right](s)=\int_{0}^{\infty} e^{B t} e^{-s t} d t=\int_{0}^{\infty} e^{(B-s) t} d t=\left[\frac{e^{(B-s) t}}{B-s}\right]_{t=0}^{t=\infty}=-\frac{1}{B-s} .
$$

In the last step we have used that

$$
\lim _{t \rightarrow \infty}\left\|\frac{e^{(B-s) t}}{B-s}\right\|_{2}=0 .
$$

Indeed, let us show (21) taking advantage of Lemma 2. On the one hand, note that by $(18) g(t)=e^{(B-s) t}$ is a 4-s.p. and, in addition, denoting $M=\sqrt[4]{c}$ and applying (19), one gets

$$
\lim _{t \rightarrow \infty}\left\|e^{(B-s) t}\right\|_{4}=\lim _{t \rightarrow \infty} e^{-s t}\left\|e^{B t}\right\|_{4} \leq M \lim _{t \rightarrow \infty} e^{(\alpha-s) t}=0 .
$$

On the other hand, we need to show that the r.v. $D=1 /(B-s) \in L_{4}$. Note that

$$
\frac{1}{B-s}=-\frac{\frac{1}{s}}{1-\frac{B}{s}}=-\frac{1}{s} \sum_{n \geq 0}\left(\frac{B}{s}\right)^{n},
$$

and taking $s>\|B\|_{4}$, the above geometric series is $m$.f. convergent and then its limit, $\frac{1}{B-s} \in L_{4}$ because $\left(L_{4},\|\cdot\|_{4}\right)$ is a Banach space. Then, by Lemma 2, from (20) one gets

$$
\mathcal{L}\left[e^{B t} H(t)\right](s)=\frac{1}{s-B}, \quad s>\max \left\{\|B\|_{4}, \alpha\right\}=\gamma
$$

This results can be extended for $s \in \mathbb{C}$. As the function $h(s)=\frac{1}{s-B}$ is an holomorphic function of the complex variable $s$ that coincides with $\mathcal{L}\left[v_{1}(t ; B)\right](s)$ in the compact set $\mathcal{K}=] \gamma, \infty[$ which has accumulation points in $\operatorname{Re}(s)>\gamma$, then by the analytic continuation principle [32, theorem 3.2.b., p.146]), expression (23) holds true for all $s$ in the half-plane $\operatorname{Re}(s)>\gamma$.

Remark 1 Condition (16) involves the computation of absolute moments of r.v. B which can be difficult because of the lack of explicit formulas even for some well-known statistical distributions. Fortunately, the Truncation Method (see [33, ch.5]) permits to obtain accurate approximations to numerous r.v.'s and it can be proven that truncated r.v.'s satisfy condition (16) (see Remark 1 in [23]). Notice that every r.v. that satisfies condition (16) has statistical moments of any order, so, in particular if $B$ satisfies condition (16), then it is a 4-r.v. and hence a 2-r.v. 
Example 2 Let $B$ be a real-valued r.v. satisfying condition (16). Let us consider the s.p.

$$
v_{2}(t ; B)=\sin (B t) H(t),
$$

where $H(t)$ denotes the Heaviside function. Then, we shall show that

$$
\mathcal{L}\left[v_{2}(t ; B)\right](s)=\frac{B}{s^{2}+B^{2}} .
$$

In fact, note that as $\sin (B t)=\operatorname{Im}\left(e^{i B t}\right)$, we consider the s.p. $e^{i B t}$. Since $B$ satisfies (16), then $i B$ also satisfies that property, see (11), and (19) holds true for $e^{i B t}$, i.e, there exists $c>0$ such that

$$
\left\|e^{i B t}\right\|_{2} \leq d e^{\alpha t}, \text { where } d=\sqrt[4]{c} .
$$

For $s \in \mathbb{R}$, one gets

$$
\begin{aligned}
\mathcal{L}\left[v_{2}(t ; B)\right](s) & =\int_{0}^{\infty} \operatorname{Im}\left(e^{i B t}\right) e^{-s t} d t=\int_{0}^{\infty} \operatorname{Im}\left(e^{i B t} e^{-s t}\right) d t \\
& =\operatorname{Im}\left(\int_{0}^{\infty} e^{i B t} e^{-s t} d t\right)=\operatorname{Im}\left(\int_{0}^{\infty} e^{(i B-s) t} d t\right),
\end{aligned}
$$

and from (20) applied to $i B$ instead of B, and using (21), one follows

$$
\begin{aligned}
\mathcal{L}\left[v_{2}(t ; B)\right](s) & =\operatorname{Im}\left(\lim _{t \rightarrow \infty}\left(\frac{e^{(i B-s) t}}{i B-s}\right)-\frac{1}{i B-s}\right)=\operatorname{Im}\left(\frac{1}{s-i B}\right) \\
& =\operatorname{Im}\left(\frac{s+i B}{(s-i B)(s+i B)}\right)=\operatorname{Im}\left(\frac{s+i B}{s^{2}+B^{2}}\right) \\
& =\frac{B}{s^{2}+B^{2}} .
\end{aligned}
$$

Notice that the limit appearing in (26) is considered in the m.s. sense. This expression can be extended for $s \in \mathbb{C}$ following an analogous reasoning we showed in the Example 1. In fact, note that the function $h(s)=\frac{B}{s^{2}+B^{2}}$ is an holomorphic function of the complex variable $s$, that coincides with $\mathcal{L}\left[v_{2}(t ; B)\right](s)$ in the compact set $\mathcal{K}=] \alpha, \infty[$ which has accumulation points in $\operatorname{Re}(s)>\alpha$. Then, by the analytic continuation principle, expression (26) holds true for all $s$ in the half-plane $\operatorname{Re}(s)>\alpha$, where $\alpha>0$ is the constant which appears in condition (16).

Example 3 Let $L$ be a r.v. satisfying condition (5), $s \in \mathbb{C}$ such that $\operatorname{Re}(s)>$ $a \geq 0$ and $x>0$. Then,

(i) $J(s)=\int_{0}^{\infty} e^{-\left(\frac{s}{z^{2}}+\frac{x^{2}}{4 L} z^{2}\right)} d z \quad$ is m.s. convergent.

(ii) $J(s)=\frac{\sqrt{\pi L}}{x} e^{-x \sqrt{\frac{s}{L}}}$ 
(iii) It is verified that

$$
\mathcal{L}\left[t^{-3 / 2} e^{-\frac{x^{2}}{4 t L}} H(t)\right](s)=\frac{2 \sqrt{\pi L}}{x} e^{-x \sqrt{\frac{s}{L}}},
$$

177

i.e., an inverse random Laplace transform of (27) is given by

$$
\mathcal{L}^{-1}\left[e^{-x \sqrt{\frac{s}{L}}}\right](t)=\frac{x}{2 \sqrt{\pi L t^{3}}} e^{-\frac{x^{2}}{4 t L}}, \quad x>0 .
$$

Let us show each of the previous statements (i)-(iii).

(i) Let $s \in \mathbb{C}$ such that $\operatorname{Re}(s)>a \geq 0$ and $x>0$ fixed,

$$
\begin{aligned}
\int_{0}^{\infty}\left\|e^{-\left(\frac{s}{z^{2}}+\frac{x^{2}}{4 L} z^{2}\right)}\right\|_{2} d z & =\int_{0}^{\infty}\left|e^{-\frac{s}{z^{2}}}\right|\left\|e^{-\frac{x^{2}}{4 L} z^{2}}\right\|_{2} d z \\
& =\int_{0}^{\infty} e^{-\frac{\operatorname{Re}(s)}{z^{2}}}\left\|e^{-\frac{x^{2}}{4 L} z^{2}}\right\|_{2} d z .
\end{aligned}
$$

From condition (5) one gets

$$
\mathrm{E}\left[\frac{1}{L^{n}}\right] \leq \frac{1}{\left(\ell_{1}\right)^{n}}, \quad n \geq 0,
$$

hence

$$
\begin{aligned}
\left(\left\|e^{-\frac{x^{2}}{4 L} z^{2}}\right\|_{2}\right)^{2} & =\mathrm{E}\left[e^{-\frac{x^{2}}{2 L} z^{2}}\right]=\sum_{n \geq 0} \frac{1}{n !}\left(\frac{-x^{2} z^{2}}{2}\right)^{n} \mathrm{E}\left[\frac{1}{L^{n}}\right] \\
& \leq \sum_{n \geq 0} \frac{1}{n !}\left(\frac{-x^{2} z^{2}}{2 \ell_{1}}\right)^{n}=e^{-\frac{x^{2} z^{2}}{2 \ell_{1}}}, \quad \forall z>0
\end{aligned}
$$

Thus,

$$
\left\|e^{-\frac{x^{2}}{4 L} z^{2}}\right\|_{2} \leq e^{-\frac{x^{2} z^{2}}{4 \ell_{1}}}, \quad \forall z>0 .
$$

From (29) and (30), and taking into account that $\operatorname{Re}(s) \geq a>0$, one gets

$$
\int_{0}^{\infty}\left\|e^{-\left(\frac{s}{z^{2}}+\frac{x^{2}}{4 L} z^{2}\right)}\right\|_{2} d z \leq \int_{0}^{\infty} e^{-\frac{\operatorname{Re}(s)}{z^{2}}} e^{-\frac{x^{2} z^{2}}{4 \ell_{1}}} d z<+\infty
$$

i.e., the integral $J(s)$ is m.s. convergent.

(ii) In part (i) we have proven that $J(s)$ is m.s. convergent and now we find a closed form expression for the s.p. $J(s)$.

Let $\omega \in \Omega$ fixed and let us consider the complex function of variable s,

$$
J(s)(\omega)=\int_{0}^{\infty} e^{-\left(\frac{s}{z^{2}}+\frac{x^{2}}{4 L(\omega)} z^{2}\right)} d z, \quad s \in \mathbb{C}: \quad \operatorname{Re}(s)>a \geq 0 .
$$


Firstly, we show that $J(\cdot)(\omega)$ is an analytic function of complex variable $s$ by using Weierstrass convergence theorem for sequences of analytic functions [34, p. 116]. Let us consider the analytic functions

$$
J_{n}(s)(\omega)=\int_{0}^{n} e^{-\left(\frac{s}{z^{2}}+\frac{x^{2}}{4 L(\omega)} z^{2}\right)} d z, \quad \operatorname{Re}(s)>a \geq 0 .
$$

Let $\mathcal{K}$ be a compact set in the open half-plane $\operatorname{Re}(s)>a \geq 0$. We wish to show that sequence $\left\{J_{n}(\cdot)(\omega): n \geq 0\right\}$ given by (32) converges uniformly in $\mathcal{K}$. Let

$$
\operatorname{Re}\left(s_{1}\right)=\min \{\operatorname{Re}(s): s \in \mathcal{K}\},
$$

then

$$
\begin{aligned}
\int_{0}^{n} \mid e^{-\left(\frac{s}{z^{2}}+\frac{x^{2}}{4 L(\omega)} z^{2}\right) \mid} d z & =\int_{0}^{n} e^{-\left(\frac{\operatorname{Re}(s)}{z^{2}}+\frac{x^{2}}{4 L(\omega)} z^{2}\right)} d z \\
& \leq \int_{0}^{n} e^{-\left(\frac{\operatorname{Re}\left(s_{1}\right)}{z^{2}}+\frac{x^{2}}{4 L(\omega)} z^{2}\right)} d z \\
& \leq \int_{0}^{\infty} e^{-\left(\frac{\operatorname{Re}\left(s_{1}\right)}{z^{2}}+\frac{x^{2}}{4 L(\omega)} z^{2}\right)} d z \\
& =\frac{\sqrt{\pi L(\omega)}}{x} e^{-x \sqrt{\frac{\operatorname{Re}\left(s_{1}\right)}{L(\omega)}}},
\end{aligned}
$$

where we have computed the last integral by [35, formula 3.325, p. 355]. Thus, $J(\cdot)(\omega)$ is an analytic function in the open half-plane $\operatorname{Re}(s)>a \geq 0$. Taking the real half-line $\mathbb{R} \cap\{s \in \mathbb{C}: \operatorname{Re}(s)>a \geq 0\}$ that has accumulation points in $\operatorname{Re}(s)>a \geq 0$, and using the value of the integral $J(s)(\omega)$ for positive real values of $s$, by the analytic continuation principle [32, theorem 3.2.b., p.146] we have that

$$
J(s)(\omega)=\frac{\sqrt{\pi L(\omega)}}{x} e^{-x \sqrt{\frac{s}{L(\omega)}}}, \quad \operatorname{Re}(s)>a \geq 0 .
$$

As (33) is true for all $\omega \in \Omega$, one gets that

$$
J(s)=\frac{\sqrt{\pi L}}{x} e^{-x \sqrt{\frac{s}{L}}} .
$$

(iii) First, let us show that the s.p.

$$
v_{3}(t ; L)=t^{-3 / 2} e^{-\frac{x^{2}}{4 t L}} H(t),
$$


is Laplace transformable. In fact, using (5)

$$
\begin{aligned}
& \left\|v_{3}(t ; L)\right\|_{2}=\frac{1}{\sqrt{t^{3}}}\left\|e^{-\frac{x^{2}}{4 t L}}\right\|_{2} \quad=\frac{1}{\sqrt{t^{3}}}\left(\mathrm{E}\left[e^{-\frac{x^{2}}{2 t L}}\right]\right)^{1 / 2} \\
& =\frac{1}{\sqrt{t^{3}}} \mathrm{E}\left[\sum_{n \geq 0} \frac{\left(\frac{-x^{2}}{2 t}\right)^{n}\left(\frac{1}{L}\right)^{n}}{n !}\right]=\frac{1}{\sqrt{t^{3}}} \sum_{n \geq 0} \frac{\left(\frac{-x^{2}}{2 t}\right)^{n} \mathrm{E}\left[\left(\frac{1}{L}\right)^{n}\right]}{n !} \\
& \leq \frac{1}{\sqrt{t^{3}}} \sum_{n \geq 0} \frac{\left(\frac{-x^{2}}{2 t}\right)^{n}\left(\frac{1}{\ell_{1}}\right)^{n}}{n !}=\frac{1}{\sqrt{t^{3}}} e^{-\frac{x^{2}}{2 t \ell_{1}}} \text {, }
\end{aligned}
$$

and

$$
\int_{0}^{\infty}\left\|v_{3}(t ; L)\right\|_{2} d t \leq \int_{0}^{\infty} \frac{1}{\sqrt{t^{3}}} e^{-\frac{x^{2}}{2 t \ell_{1}}} e^{-s t} d t<\infty, \quad s \in \mathbb{C}: \quad \operatorname{Re}(s)>0 .
$$

Using the definition of random Laplace transform, doing a suitable change of variable and using (ii) one gets

$$
\begin{aligned}
\mathcal{L}\left[t^{-3 / 2} e^{-\frac{x^{2}}{4 t L}} H(t)\right](s) & =\int_{0}^{\infty} \frac{e^{-\frac{x^{2}}{4 t L}}}{t^{3 / 2}} e^{-s t} d t=\left[\frac{1}{\sqrt{t}}=z\right] \\
& =2 \int_{0}^{\infty} e^{-\left(\frac{s}{z^{2}}+\frac{x^{2}}{4 L} z^{2}\right)} d z \\
& =2 J(s)=\frac{2 \sqrt{\pi L}}{x} e^{-x \sqrt{\frac{s}{L}}}, \quad x>0 .
\end{aligned}
$$

\subsection{Operational rules for random Laplace transform}

Let $u(t)$ be a 2-s.p. m.s. differentiable such as that $u^{\prime}(t)$ is m.s. continuous and both, $u(t)$ and $u^{\prime}(t)$, belong to the class $\mathfrak{C}$. Assume that the 2-s.p. $u(t)$ exists at the right of zero, that is, exists $u(0+)=\lim _{t \rightarrow 0^{+}} u(t)$. Then from definition (14) and using the fundamental theorem of m.s. calculus, [19, p. 104], one gets

$$
\begin{aligned}
\mathcal{L}\left[u^{\prime}(t)\right](s) & =\int_{0}^{\infty} u^{\prime}(t) e^{-s t} d t=\left[u(t) e^{-s t}\right]_{t=0}^{t=\infty}+s \int_{0}^{\infty} u(t) e^{-s t} d t \\
& =\left[u(t) e^{-s t}\right]_{t=0}^{t=\infty}+s \mathcal{L}[u(t)](s) .
\end{aligned}
$$

Now, by applying condition (13) to $u(t)$ and taking $\operatorname{Re}(s)>a$, it is verified

$$
\left\|u(t) e^{-s t}\right\|_{2}=\left|e^{-s t}\right|\|u(t)\|_{2} \leq M e^{-t \operatorname{Re}(s)} e^{a t}=M e^{t(a-\operatorname{Re}(s))} \stackrel{t \rightarrow+\infty}{\longrightarrow} 0 .
$$

Then, from (35)-(36) it is obtained the following operational rule which relates the random Laplace transform of a 2-s.p. with the random Laplace transform of its first m.s. derivative

$$
\mathcal{L}\left[u^{\prime}(t)\right](s)=s \mathcal{L}[u(t)](s)-u(0+) .
$$


The next operational rule is the convolution for 2-s.p.'s $f(t)$ and $g(t)$ of class $\mathfrak{C}$, denoted by $f * g$ and defined by the m.s. integral

$$
(f * g)(t)=\int_{0}^{t} f(t-\nu) g(\nu) d \nu, \quad t \geq 0 .
$$

As it occurs for the deterministic case, see [36, p. 259] by writing $\mathcal{L}[f * g]$ as a double m.s. integral and reversing the order of integration, one gets a random convolution formula for the random Laplace transform

$$
\mathcal{L}[f * g](s)=\mathcal{L}[f](s) \mathcal{L}[g](s)=F(s) G(s), \quad f, g \in \mathfrak{C} .
$$

\section{Random heat problem}

This section deals with the construction of the solution s.p. of the problem (1)(4) as well as the determination of its expectation and variance. Let us assume that $L$ is a positive r.v. that satisfies condition $(5)$, and let $f(t ; A)$ be a s.p. in the class $\mathfrak{C}$. Assume that the problem (1)-(4) admits a Laplace transformable solution s.p. $u(x, t)$ which will be denoted by

$$
\mathcal{L}[u(x, \cdot)](s)=\mathcal{U}(x)(s), \quad s \in \mathbb{C}: \operatorname{Re}(s)>a \geq 0,
$$

what means that $u(x, t)$ is regarded as a s.p. of the active variable $t>0$, for fixed $x>0$. Now, we apply the random Laplace transform to both members of equation (1). For the left-hand side, we use property (37) and the initial condition (2), this yields

$$
\mathcal{L}\left[u_{t}(x, \cdot)\right](s)=s \mathcal{U}(x)(s)-u(x, 0+)=s \mathcal{U}(x)(s),
$$

and, for the right-hand side, we apply twice Lemma 2 of [23]

$$
\mathcal{L}\left[u_{x x}(x, \cdot)\right](s)=\int_{0}^{\infty} u_{x x}(x, \cdot) e^{-s t} d t=\frac{d^{2} \mathcal{U}(x)(s)}{d x^{2}} .
$$

By applying the random Laplace transform to conditions (3) and (4), it follows that

$$
\mathcal{U}(0)(s)=\mathcal{L}[u(0, \cdot)](s)=\mathcal{L}[f(\cdot ; A)](s)=F(s ; A),
$$

and

$$
\mathcal{U}(x)(s)=\mathcal{L}[u(x, \cdot)](s) \text { is bounded if } x \rightarrow+\infty .
$$

Hence, the problem (1)-(4) has been transformed into the following random initial value problem based on a second-order differential equation

$$
\begin{aligned}
& \frac{d^{2}}{d x^{2}} \mathcal{U}(x)(s)-\frac{s}{L} \mathcal{U}(x)(s)=0, \quad x>0, \\
& \mathcal{U}(0)(s)=F(s ; A), \\
& \mathcal{U}(x)(s)=\mathcal{L}[u(x, \cdot)](s) \quad \text { is bounded if } x \rightarrow+\infty .
\end{aligned}
$$


In accordance with Proposition 9 of [37], the set $\left\{e^{x \sqrt{s / L}}, e^{-x \sqrt{s / L}}\right\}$ is a fundamental system of solutions of the problem $(41)-(43)$, since as $\operatorname{Re}(s)>0$ and $L$ satisfies condition (5), its Wronskian, $-2 \sqrt{\frac{s}{L}}$, is well-defined and different from zero for all $\omega \in \Omega$. Then, a general solution s.p. of the random ordinary differential equation (41) is given by

$$
\mathcal{U}(x)(s)=C_{1}(s) e^{x \sqrt{s / L}}+C_{2}(s) e^{-x \sqrt{s / L}} .
$$

Taking into account condition (43), we put $C_{1}(s)=0$, thus from (44) we seek a solution s.p. of the form

$$
\mathcal{U}(x)(s)=C_{2}(s) e^{-x \sqrt{s / L}},
$$

which applying condition (42) takes the form

$$
\mathcal{U}(x)(s)=F(s ; A) e^{-x \sqrt{s / L}}=F(s ; A) \mathcal{L}[g(t ; L)](s),
$$

where, by (iii) of Example 3, the s.p. $g(t ; L)$ takes the form

$$
g(t ; L)=\mathcal{L}^{-1}\left[e^{-x \sqrt{s / L}}\right](t)=\frac{x}{2 \sqrt{\pi L t^{3}}} e^{-x^{2} / 4 t L} .
$$

Then, by taking the random inverse Laplace transform in (46), considering the convolution property (39) and using (38) and (47), one gets a solution 2-s.p. of problem (1)-(4):

$$
\begin{aligned}
u(x, t) & =\mathcal{L}^{-1}[\mathcal{U}(x)(s)](t)=\mathcal{L}^{-1}\left[F(s ; A) e^{-x \sqrt{s / L}}\right](t) \\
& =\mathcal{L}^{-1}[\mathcal{L}[(f * g)(t ; A, L)](s)](t) \\
& =(f * g)(t ; A, L)=\int_{0}^{t} f(t-\nu ; A) g(\nu ; L) d \nu \\
& =\frac{x}{2 \sqrt{\pi L}} \int_{0}^{t} \frac{e^{-x^{2} / 4 \nu L}}{\sqrt{\nu^{3}}} f(t-\nu ; A) d \nu, \quad x>0, \quad t>0 .
\end{aligned}
$$

Summarizing, the following result has been established

Theorem 1 Let us consider the random heat problem (1)-(4) where $L$ is a positive r.v. satisfying condition (5), and let $f(t ; A)$ be a s.p. in the class $\mathfrak{C}$ which depends on r.v. A. Then, the m.s. solution s.p. $u(x, t)$ of problem (1)-(4) is given by (48) .

Assuming independence of r.v.'s $L$ and $A$ the expectation and the variance functions of the solution s.p. $u(x, t)$, given by (47), can be computed by the following closed expressions:

$$
\mathrm{E}[u(x, t)]=\frac{x}{2 \sqrt{\pi}} \int_{0}^{t} \mathrm{E}\left[\frac{1}{\sqrt{L \nu^{3}}} e^{-x^{2} / 4 \nu L}\right] \mathrm{E}[f(t-\nu ; A)] d \nu,
$$




$$
\operatorname{Var}[u(x, t)]=\mathrm{E}\left[(u(x, t))^{2}\right]-(\mathrm{E}[u(x, t)])^{2},
$$

where

$$
\begin{aligned}
& \mathrm{E}\left[(u(x, t))^{2}\right] \\
= & \frac{x^{2}}{4 \pi} \int_{0}^{t} \int_{0}^{t} \mathrm{E}\left[\frac{1}{L \sqrt{\left(\nu_{1}\right)^{3}\left(\nu_{2}\right)^{3}}} e^{-\frac{x^{2}\left(\nu_{1}+\nu_{2}\right)}{4 \nu_{1} \nu_{2} L}}\right] \mathrm{E}\left[f\left(t-\nu_{1} ; A\right) f\left(t-\nu_{2} ; A\right)\right] d \nu_{1} d \nu_{2} .
\end{aligned}
$$

If $g_{L}(l)$ and $g_{A}(a)$ denote the p.d.f.'s of the random inputs $L$ and $A$, and $\mathcal{D}(L)$ and $\mathcal{D}(A)$ denote their domains, respectively, then taking into account (12) the expectations that appear in the above integrals can be computed as follows

$$
\mathrm{E}\left[\frac{1}{\sqrt{L \nu^{3}}} e^{-x^{2} / 4 \nu L}\right]=\int_{\mathcal{D}(L)} \frac{1}{\sqrt{L \nu^{3}}} e^{-x^{2} / 4 \nu L} g_{L}(l) d l,
$$

$$
\mathrm{E}[f(t-\nu ; A)]=\int_{\mathcal{D}(A)} f(t-\nu ; a) g_{A}(a) d a,
$$

$$
\mathrm{E}\left[\frac{1}{L \sqrt{\left(\nu_{1}\right)^{3}\left(\nu_{2}\right)^{3}}} e^{-\frac{x^{2}\left(\nu_{1}+\nu_{2}\right)}{4 \nu_{1} \nu_{2} L}}\right]=\int_{\mathcal{D}(L)} \frac{1}{L \sqrt{\left(\nu_{1}\right)^{3}\left(\nu_{2}\right)^{3}}} e^{-\frac{x^{2}\left(\nu_{1}+\nu_{2}\right)}{4 \nu_{1} \nu_{2} l}} g_{L}(l) d l,
$$

$$
\mathrm{E}\left[f\left(t-\nu_{1} ; A\right) f\left(t-\nu_{2} ; A\right)\right]=\int_{\mathcal{D}(A)} f\left(t-\nu_{1} ; a\right) f\left(t-\nu_{2} ; a\right) g_{A}(a) d a .
$$

These expressions permit to understand that the expectation and the variance of the solution s.p. $u(x, t)$ get modified by different choice of p.d.f.'s of random input parameters $L$ and $A$ in practice.

\section{$5 \quad$ Numerical examples}

In this section, we illustrate the theorical results previously developed by means of a numerical example where the expectation and the variance to the solution s.p. $u(x, t)$, given by $(49)-(55)$ are computed. Computations have been carried out using the software Mathematica ${ }^{\circledR}$.

Example 4 Let us consider the mixed random parabolic problem (1)-(4) where the random diffusion coefficient $L$ is assumed to be a positive r.v. which has a truncated gamma distribution of parameters $\alpha=3$ and $\beta=2$, i.e., $L \sim$ $G a(3 ; 2)$, on the interval $[0.1,3]$. Hence, $L$ satisfies condition $(5)$. Let $f(t ; A)=$ $e^{A t} H(t)$ be a s.p. depending on r.v. A which is assumed to have a beta distribution of parameters $\alpha=2$ and $\beta=1$, i.e. $A \sim B e(2 ; 1)$. Since $A$ is by its own definition truncated, then condition (16) is satisfied and according to Example 1, $f(t ; A)$ is in the class $\mathfrak{C}$. Therefore, hypotheses of Theorem (1) hold true and the m.s. solution stochastic process, $u(x, t)$, to problem (1)-(4) is given by (48). 
Assuming that $L$ and $A$ are independent r.v.'s, the expectation and the variance of $u(x, t)$ can be exactly computed by expressions (49)-(55). Figure 1 shows the evolution of average temperature (plot (a)) on a bar of length $0 \leq x \leq 5$ at different time instants as well as its variation measured through the standard deviation (plot (b)). Since average temperature tends to increase (decrease) at the left-end (right-end) of the bar as times goes on, the variability behaves in the same manner. For the sake of clarity, in Figure 2 we show this behaviour in 3-D over a longer time interval.

In Figures 3 and 4, we compare the exact values of the expectation and the standard deviation, respectively, against the ones obtained by Monte Carlo sampling using 100, 500 and 1000 simulations at the time instants $t \in\{0.4,0.6,0.8,1\}$ on the piece $] 0,3]$ of the spatial domain, $x \in] 0,5]$. In order to complete this analysis, in Tables 1-2 we have collected the exact values of the mean, $\mathrm{E}\left[u\left(x_{i}, t\right)\right]$, and, standard deviation, $\sqrt{\operatorname{Var}\left[u\left(x_{i}, t\right)\right]}$, at different spatial values $x_{i} \in\{0.1,0.5,1,1.5,2,2.5,3,3.5,4,4.5,5\}$ at the times instants $t=0.5$ and $t=2$, respectively. The corresponding values obtained by Monte Carlo method using $r=10^{2}, r=5 \times 10^{2}, r=10^{3}$ and $r=10^{4}$ have been included too. In order to account for the quality of Monte Carlo results, the values of the relative errors for the mean, RelErr $\mu_{\mu_{r} M C}$, and the standard deviation, RelErr ${ }_{\sigma_{r} M C}$, using $r$ Monte Carlo simulations have been also computed according to the following expressions

$$
\begin{aligned}
\operatorname{RelErr}_{\mu_{r} M C} & =\left|\frac{\mathrm{E}\left[u\left(x_{i}, t\right)\right]-\mu_{r} M C\left(x_{i}, t\right)}{\mathrm{E}\left[u\left(x_{i}, t\right)\right]}\right|, \\
\operatorname{RelErr}_{\sigma_{r} M C} & =\left|\frac{\sqrt{\operatorname{Var}\left[u\left(x_{i}, t\right)\right]}-\sigma_{r} M C\left(x_{i}, t\right)}{\sqrt{\operatorname{Var}\left[u\left(x_{i}, t\right)\right]}}\right| .
\end{aligned}
$$

The consistency of the estimation of the moments is clearly manifested since the numerical results via Monte Carlo are closer to the exact ones obtained by the proposed random mean square approach by (49)-(55), as the number $r$ of simulations increases. Monte Carlo simulations were carried out by Mathematica ${ }^{\circledR}$ software version 10 for Linux x86 (64-bit) using 32 Xeon-double-processors with half-terabyte capacity. Regarding computational time, figures collected in Table 1 for $r=10^{4}$ simulations by Monte Carlo required 86 minutes and 17 seconds. Timing was similar for the same computations shown in Table 2. Whereas 15 hours, 28 minutes and 16 seconds were needed to compute analogous approximations with spatial 50 points $x_{i}$ instead of 11 spatial points. These timings are higher than the ones needed using our random mean square approach, whose execution time was a few seconds. Parallelization was used to carry out computations using both approaches. 


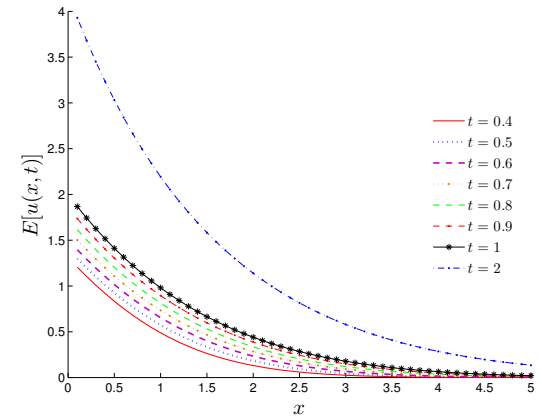

(a)

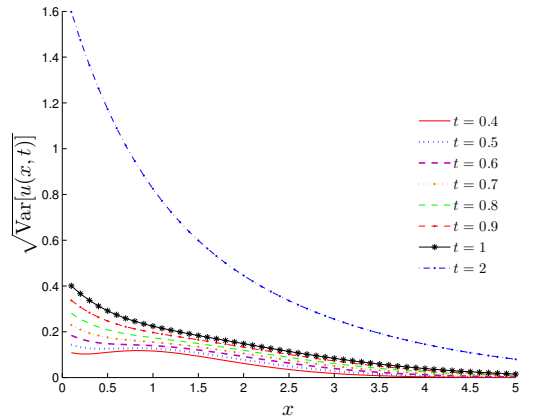

(b)

Figure 1: Evolution of the expectation $\mathrm{E}[u(x, t)]$ (plot (a)), and the standard deviation $\sqrt{\operatorname{Var}[w(x, t)]}$ (plot (b)) on the spatial domain $x \in] 0,5]$ at different time instants in the context of Example 4.

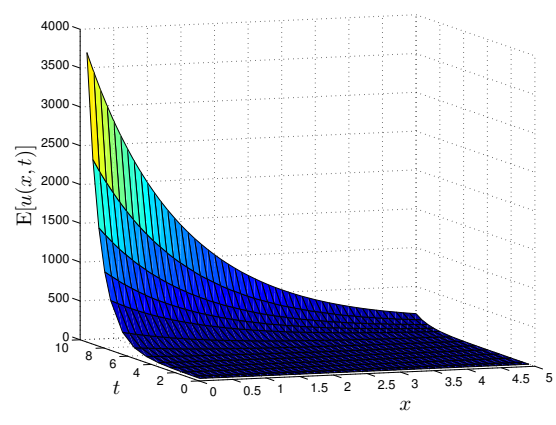

(a)

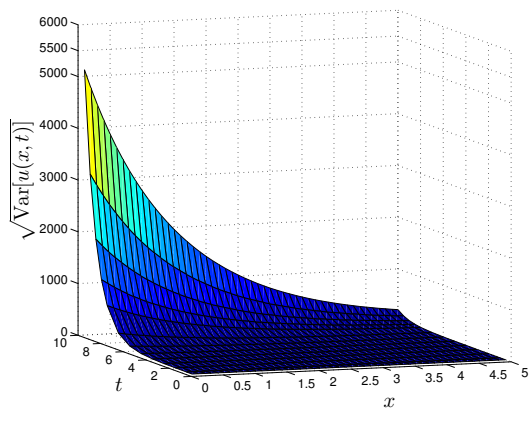

(b)

Figure 2: Three-dimensional approximations for the evolution of the expectation $\mathrm{E}[u(x, t)]$ (plot (a)), and, the standard deviation $\sqrt{\operatorname{Var}[w(x, t)]}$ (plot (b)) on the spatial domain $x \in] 0,5]$ throughout the time interval $t \in] 0,10]$ in the context of Example 4. 


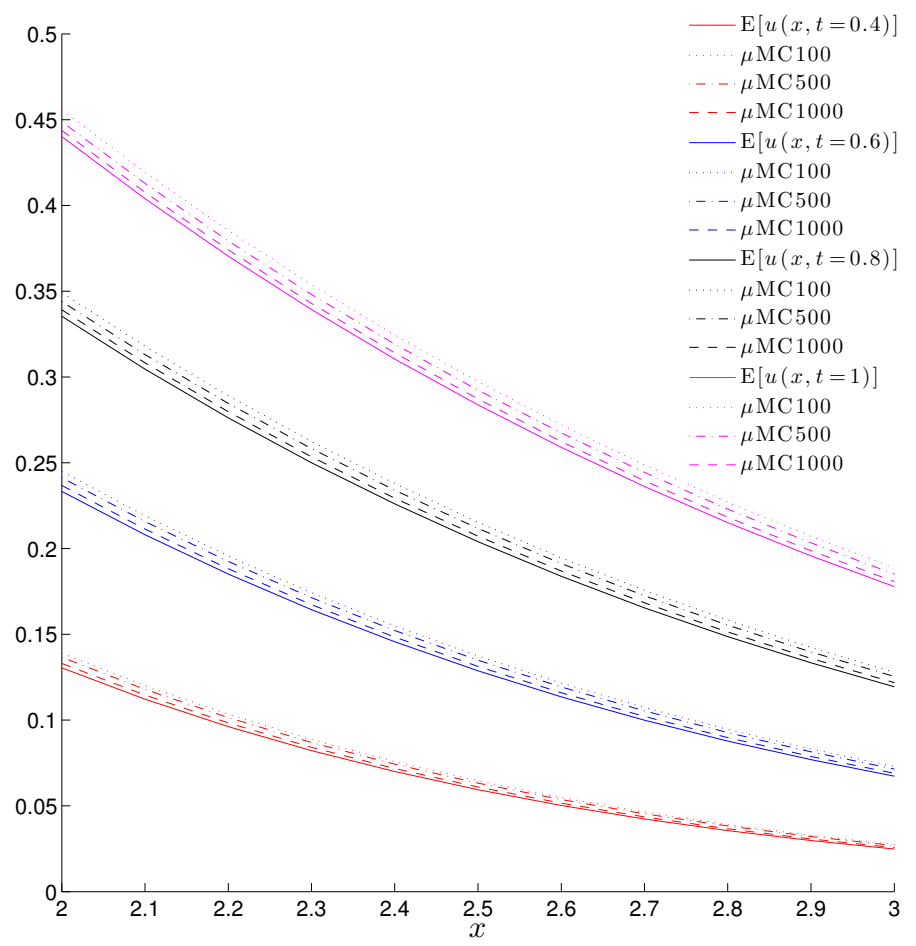

Figure 3: Comparison between the exact values of the expectation of the solution, $\mathrm{E}[u(x, t)]$, given by (49), (52)-(53), and Monte Carlo ( $\mu \mathrm{MC})$ using 100, 500 and 1000 simulations at the time instants $t=0.4, t=0.6, t=0.8$ and $t=1$ on the piece $] 0,3]$ of spatial domain, $x \in] 0,5]$. 


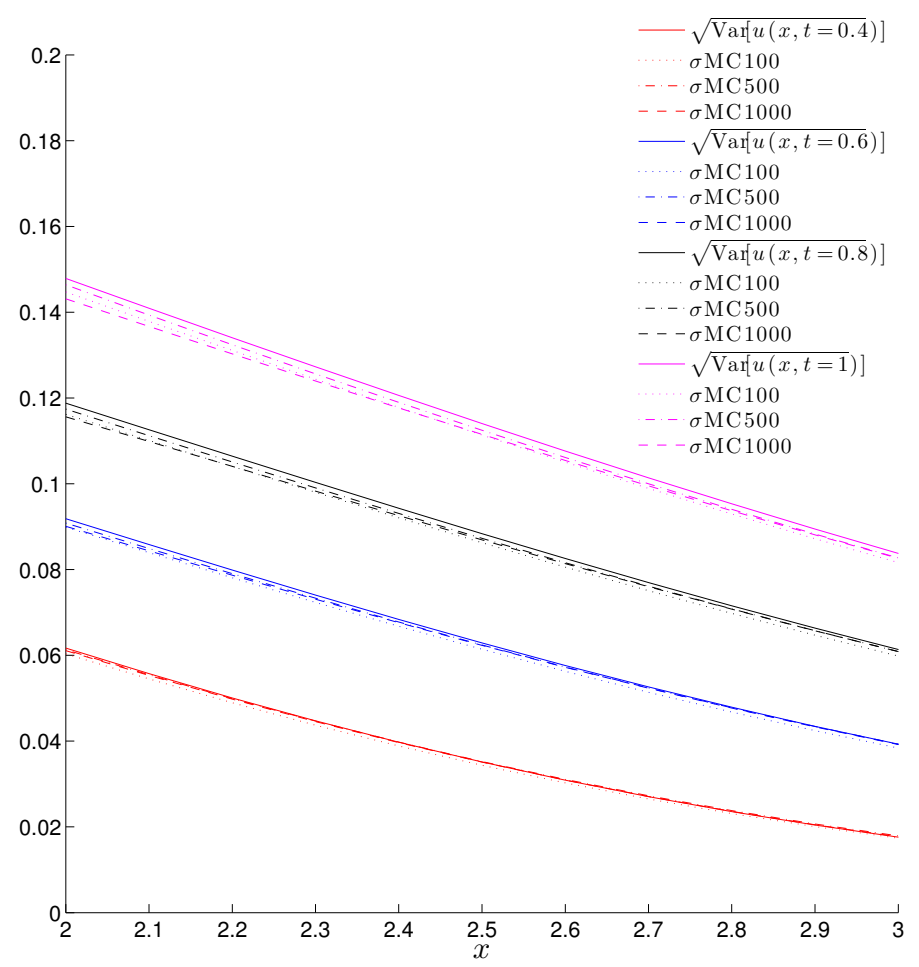

Figure 4: Comparison between the exact values of the standard deviation of the solution, $\sqrt{\operatorname{Var}[u(x, t)]}$, given by (49)-(55), and Monte Carlo $(\sigma \mathrm{MC})$ using 100 , 500 and 1000 simulations at the time instants $t=0.4, t=0.6, t=0.8$ and $t=1$ on the piece $] 0,3]$ of spatial domain, $x \in] 0,5]$. 


\begin{tabular}{|c|c|c|c|c|c|c|c|c|c|c|c|c|}
\hline \multirow[t]{2}{*}{$\bar{t}=0.5$} & & \multicolumn{11}{|c|}{$x_{i}$} \\
\hline & $r$ & 0.1 & 0.5 & 1.0 & 1.5 & 2.0 & 2.5 & 3.0 & 3.5 & 4.0 & 4.5 & 5.0 \\
\hline $\mathrm{E}\left[u\left(x_{i}, t\right)\right]$ & & $1.2982 \mathrm{e}+00$ & $9.2504 \mathrm{e}-01$ & $5.7356 \mathrm{e}-01$ & $3.3380 \mathrm{e}-01$ & $1.8207 \mathrm{e}-01$ & $9.2991 \mathrm{e}-02$ & $4.4432 \mathrm{e}-02$ & $1.9843 \mathrm{e}-02$ & $8.2747 \mathrm{e}-03$ & $3.2188 \mathrm{e}-03$ & $1.1667 \mathrm{e}-03$ \\
\hline & $10^{2}$ & $1.2994 \mathrm{e}+00$ & $9.3381 \mathrm{e}-01$ & $5.8637 \mathrm{e}-01$ & $3.4654 \mathrm{e}-01$ & $1.9237 \mathrm{e}-01$ & $1.0004 \mathrm{e}-01$ & $4.8597 \mathrm{e}-02$ & $2.2009 \mathrm{e}-02$ & $9.2819 \mathrm{e}-03$ & $3.6425 \mathrm{e}-03$ & $1.3296 \mathrm{e}-03$ \\
\hline$\mu_{r} \operatorname{MC}\left(x_{i}, t\right)$ & $5 \times 10^{2}$ & $1.2917 \mathrm{e}+00$ & $9.2575 \mathrm{e}-01$ & $5.8005 \mathrm{e}-01$ & $3.4211 \mathrm{e}-01$ & $1.8934 \mathrm{e}-01$ & $9.8128 \mathrm{e}-02$ & $4.7564 \mathrm{e}-02$ & $2.1542 \mathrm{e}-02$ & $9.1075 \mathrm{e}-03$ & $3.5911 \mathrm{e}-03$ & $1.3193 \mathrm{e}-03$ \\
\hline & $10^{3}$ & $1.2943 \mathrm{e}+00$ & $9.2603 \mathrm{e}-01$ & $5.7726 \mathrm{e}-01$ & $3.3776 \mathrm{e}-01$ & $1.8520 \mathrm{e}-01$ & $9.5090 \mathrm{e}-02$ & $4.5682 \mathrm{e}-02$ & $2.0515 \mathrm{e}-02$ & $8.6027 \mathrm{e}-03$ & $3.3646 \mathrm{e}-03$ & $1.2260 \mathrm{e}-03$ \\
\hline & $10^{4}$ & $1.2969 \mathrm{e}+00$ & $9.2494 \mathrm{e}-01$ & $5.7378 \mathrm{e}-01$ & $3.3391 \mathrm{e}-01$ & $1.8210 \mathrm{e}-01$ & $9.3003 \mathrm{e}-02$ & $4.4455 \mathrm{e}-02$ & $1.9869 \mathrm{e}-02$ & $8.2953 \mathrm{e}-03$ & $3.2313 \mathrm{e}-03$ & $1.1732 \mathrm{e}-03$ \\
\hline & $10^{2}$ & $9.2009 \mathrm{e}-04$ & $9.4735 \mathrm{e}-03$ & $2.2345 \mathrm{e}-02$ & $3.8161 \mathrm{e}-02$ & $5.6582 \mathrm{e}-02$ & $7.5781 \mathrm{e}-02$ & $9.3725 \mathrm{e}-02$ & $1.0916 \mathrm{e}-01$ & $1.2172 \mathrm{e}-01$ & $1.3166 \mathrm{e}-01$ & $1.3961 \mathrm{e}-01$ \\
\hline $\operatorname{RelErr}_{\mu_{r} \mathrm{MC}}$ & $5 \times 10^{2}$ & $4.9847 \mathrm{e}-03$ & $7.6350 \mathrm{e}-04$ & $1.1322 \mathrm{e}-02$ & $2.4912 \mathrm{e}-02$ & $3.9923 \mathrm{e}-02$ & $5.5251 \mathrm{e}-02$ & $7.0487 \mathrm{e}-02$ & $8.5598 \mathrm{e}-02$ & $1.0064 \mathrm{e}-01$ & $1.1569 \mathrm{e}-01$ & $1.3077 \mathrm{e}-01$ \\
\hline & $10^{3}$ & $2.9926 \mathrm{e}-03$ & $1.0602 \mathrm{e}-03$ & $6.4636 \mathrm{e}-03$ & $1.1884 \mathrm{e}-02$ & $1.7207 \mathrm{e}-02$ & $2.2572 \mathrm{e}-02$ & $2.8120 \mathrm{e}-02$ & $3.3854 \mathrm{e}-02$ & $3.9636 \mathrm{e}-02$ & $4.5320 \mathrm{e}-02$ & $5.0799 \mathrm{e}-02$ \\
\hline & $10^{4}$ & $1.0166 \mathrm{e}-03$ & $1.1824 \mathrm{e}-04$ & $3.8882 \mathrm{e}-04$ & $3.4693 \mathrm{e}-04$ & $1.4106 \mathrm{e}-04$ & $1.3129 \mathrm{e}-04$ & $5.1466 \mathrm{e}-04$ & $1.3262 \mathrm{e}-03$ & $2.4844 \mathrm{e}-03$ & $3.9047 \mathrm{e}-03$ & $5.5091 \mathrm{e}-03$ \\
\hline$\sqrt{\operatorname{Var}\left[u\left(x_{i}, t\right)\right]}$ & & $1.4371 \mathrm{e}-01$ & $1.2577 \mathrm{e}-01$ & $1.2666 \mathrm{e}-01$ & $1.0741 \mathrm{e}-01$ & $7.7609 \mathrm{e}-02$ & $4.9405 \mathrm{e}-02$ & $2.8263 \mathrm{e}-02$ & $1.4694 \mathrm{e}-02$ & $6.9892 \mathrm{e}-03$ & $3.0533 \mathrm{e}-03$ & $1.2280 \mathrm{e}-03$ \\
\hline \multirow[t]{5}{*}{$\vec{\infty}$} & $10^{2}$ & $1.4819 \mathrm{e}-01$ & $1.2238 \mathrm{e}-01$ & $1.2327 \mathrm{e}-01$ & $1.0531 \mathrm{e}-01$ & $7.5940 \mathrm{e}-02$ & $4.8260 \mathrm{e}-02$ & $2.7695 \mathrm{e}-02$ & $1.4513 \mathrm{e}-02$ & $6.9804 \mathrm{e}-03$ & $3.0891 \mathrm{e}-03$ & $1.2594 \mathrm{e}-03$ \\
\hline & $5 \times 10^{2}$ & $1.4688 \mathrm{e}-01$ & $1.2882 \mathrm{e}-01$ & $1.2761 \mathrm{e}-01$ & $1.0659 \mathrm{e}-01$ & $7.6854 \mathrm{e}-02$ & $4.9242 \mathrm{e}-02$ & $2.8459 \mathrm{e}-02$ & $1.4964 \mathrm{e}-02$ & $7.1971 \mathrm{e}-03$ & $3.1770 \mathrm{e}-03$ & $1.2900 \mathrm{e}-03$ \\
\hline & $10^{3}$ & $1.3886 \mathrm{e}-01$ & $1.1906 \mathrm{e}-01$ & $1.2125 \mathrm{e}-01$ & $1.0444 \mathrm{e}-01$ & $7.6457 \mathrm{e}-02$ & $4.9148 \mathrm{e}-02$ & $2.8309 \mathrm{e}-02$ & $1.4791 \mathrm{e}-02$ & $7.0622 \mathrm{e}-03$ & $3.0955 \mathrm{e}-03$ & $1.2488 \mathrm{e}-03$ \\
\hline & $10^{4}$ & $1.4371 \mathrm{e}-01$ & $1.2359 \mathrm{e}-01$ & $1.2462 \mathrm{e}-01$ & $1.0653 \mathrm{e}-01$ & $7.7413 \mathrm{e}-02$ & $4.9434 \mathrm{e}-02$ & $2.8319 \mathrm{e}-02$ & $1.4730 \mathrm{e}-02$ & $7.0073 \mathrm{e}-03$ & $3.0615 \mathrm{e}-03$ & $1.2316 \mathrm{e}-03$ \\
\hline & $10^{2}$ & $3.1170 \mathrm{e}-02$ & $2.6951 \mathrm{e}-02$ & $2.6796 \mathrm{e}-02$ & $1.9534 \mathrm{e}-02$ & $2.1514 \mathrm{e}-02$ & $2.3178 \mathrm{e}-02$ & $2.0086 \mathrm{e}-02$ & $1.2316 \mathrm{e}-02$ & $1.2544 \mathrm{e}-03$ & $1.1750 \mathrm{e}-02$ & $2.5612 \mathrm{e}-02$ \\
\hline \multirow[t]{3}{*}{$\operatorname{RelErr}_{\sigma_{r} \mathrm{MC}}$} & $5 \times 10^{2}$ & $2.2010 \mathrm{e}-02$ & $2.4316 \mathrm{e}-02$ & $7.5127 \mathrm{e}-03$ & $7.6383 \mathrm{e}-03$ & $9.7360 \mathrm{e}-03$ & $3.3188 \mathrm{e}-03$ & $6.9199 \mathrm{e}-03$ & $1.8356 \mathrm{e}-02$ & $2.9750 \mathrm{e}-02$ & $4.0547 \mathrm{e}-02$ & $5.0529 \mathrm{e}-02$ \\
\hline & $10^{3}$ & $3.3774 \mathrm{e}-02$ & $5.3328 \mathrm{e}-02$ & $4.2744 \mathrm{e}-02$ & $2.7632 \mathrm{e}-02$ & $1.4849 \mathrm{e}-02$ & $5.2154 \mathrm{e}-03$ & $1.6261 \mathrm{e}-03$ & $6.5554 \mathrm{e}-03$ & $1.0444 \mathrm{e}-02$ & $1.3838 \mathrm{e}-02$ & $1.6921 \mathrm{e}-02$ \\
\hline & $10^{4}$ & $2.8036 \mathrm{e}-06$ & $1.7344 \mathrm{e}-02$ & $1.6073 \mathrm{e}-02$ & $8.1444 \mathrm{e}-03$ & $2.5332 \mathrm{e}-03$ & $5.6927 \mathrm{e}-04$ & $1.9644 \mathrm{e}-03$ & $2.4453 \mathrm{e}-03$ & $2.5864 \mathrm{e}-03$ & $2.7164 \mathrm{e}-03$ & $2.9593 \mathrm{e}-03$ \\
\hline
\end{tabular}

Table 1: Values of the exact expectation, $\mathrm{E}\left[u\left(x_{i}, t\right)\right]$, and standard deviation, $\sqrt{\operatorname{Var}\left[u\left(x_{i}, t\right)\right]}$, given by $(49)-(55)$, at some spatial points $\left.\left.x_{i} \in\right] 0,5\right]$ at the time instant $t=0.5$ for Example 4 . The values of the mean and the standard deviation obtained by Monte Carlo, $\mu_{r} \mathrm{MC}\left(x_{i}, t\right)$, and $\sigma_{r} \mathrm{MC}\left(x_{i}, t\right)$, respectively, using $r=10^{2}, r=5 \times 10^{2}, r=10^{3}$ and $r=10^{4}$ simulations are shown too. The comparison between the values of the mean and the standard deviation obtained using both methods are made by considering the relative errors in each $x_{i}$ for each number $r$ of simulations according to (56). 


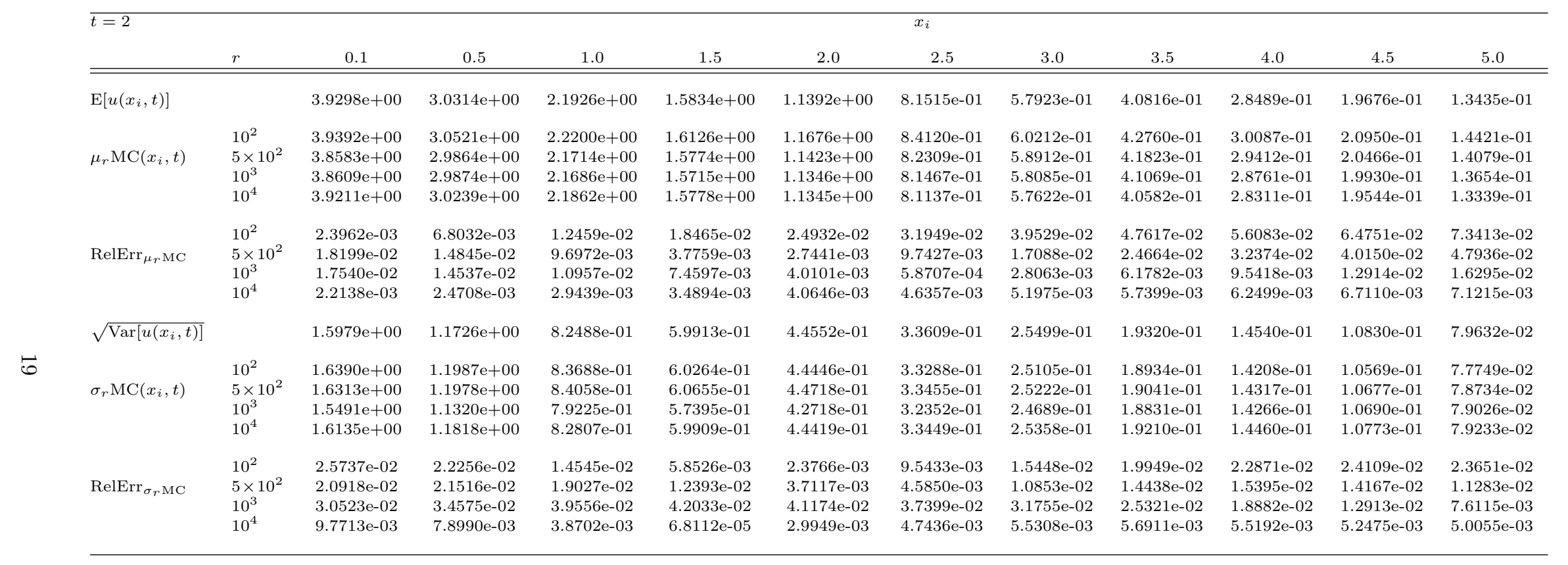

Table 2: Values of the exact expectation, $\mathrm{E}\left[u\left(x_{i}, t\right)\right]$, and standard deviation, $\sqrt{\operatorname{Var}\left[u\left(x_{i}, t\right)\right]}$, given by $(49)-(55)$, at some spatial points $\left.\left.x_{i} \in\right] 0,5\right]$ at the time instant $t=2$ for Example 4 . The values of the mean and the standard deviation obtained by Monte Carlo, $\mu_{r} \mathrm{MC}\left(x_{i}, t\right)$, and $\sigma_{r} \mathrm{MC}\left(x_{i}, t\right)$, respectively, using $r=10^{2}, r=5 \times 10^{2}, r=10^{3}$ and $r=10^{4}$ simulations are shown too. The comparison between the values of the mean and the standard deviation obtained using both methods are made by considering the relative errors in each $x_{i}$ for each number $r$ of simulations according to (56). 


\section{Conclusions}

In this paper, we have first introduced the random Laplace transform of an stochastic process in the mean square probabilistic sense including several illustrative examples where the Laplace transform is computed. The classical definition of original function is extended for original stochastic processes and the hypothesis of growth not greater than an exponential is replaced by the growth of the mean square norm of the stochastic process. Secondly, after introducing some operational calculus for the random Laplace transform, we show the capability of this random transform to obtain a closed-form solution stochastic process of the mixed partial differential problem (1)-(4). The obtained theoretical results are illustrated by means of an example where the expectation and the variance of the solution s.p. are computed. We emphasize that the proposed approach can be applied to deal with other problems based on mixed partial differential equations which often appear in physical models as well as to extend to the random scenario further classical transforms that have demonstrated to be useful tools to solve partial differential problems.

\section{Acknowledgements}

This work has been partially supported by the Spanish Ministerio de Economía y Competitividad grant MTM2013-41765-P and by the European Union in the FP7-PEOPLE-2012-ITN Program under Grant Agreement no. 304617 (FP7 Marie Curie Action, Project Multi-ITN STRIKE-Novel Methods in Computational Finance).

\section{References}

[1] Farlow, Partial Differntial Equations, Dover Publications, Inc., New York, 1968.

[2] M.N. Özişik, Boundary Value Problems of Heat Conduction, Dover Publications, Inc., New York, 1968.

[3] G.B. Davis, A Laplace transform technique for the analytical solution of a diffusion- convection equation over a finite domain, Appl. Math. Modelling, 9 (1985) 69-71.

[4] T. Myint-U, L. Debnath, Partial Differential Equations for Scientists and Engineers, third edition, Elsevier, New York, 1987.

[5] L. Debnath, Integral transforms and their applications, CRC Press, New York, 1995.

[6] E. Momoniat, R. McIntyre, R. Ravindran, Numerical inversion of a Laplace transform solution of a diffusion equation with a mixed derivative term, Appl. Math. Comput., 209 (2) (2009) 222-229. 
[7] A.-M. Wazwaz, The combined Laplace transform-Adomian decomposition method for handling nonlinear Volterra integro-differential equations, Appl. Math. Comput., 216 (4) (2010) 1304-1309.

[8] Y. Khan, A novel Laplace decomposition method for non-linear stretching sheet problem in the presence of MHD and slip condition, Int. J. Numer. Method H., 24 (1) (2014) 73-85.

[9] S. Xiang, Laplace transforms for approximation of highly oscillatory Volterra integral equations of the first kind, Appl. Math. Comput., 232 (2014) 944-954.

[10] G. Dagan, S.P. Neuman, Subsurface Flow and Transport: A Stochastic Approach, Cambridge University Press, Cambridge, 1997.

[11] Y. Rubin, Applied Stochastic Hydrogeology, Oxford University Press, Oxford, 2003.

[12] D. Zhang, Stochastic Methods for Flow in Porous Media: Coping With Uncertainties, Elsevier, 2002.

[13] D. M. Tartakovsky, S. P. Neuman, Transient flow in bounded randomly heterogeneous domains. 1. Exact conditional moment equations and recursive approximations, Water Resour. Res., 34(1) (1998) 1-12.

[14] D. M. Tartakovsky, S. P. Neuman, Transient effective hydraulic conductivities under slowly and rapidly varying mean gradients in bounded threedimensional random media, Water Resour. Res., 34(1) (1998) 21-32.

[15] D.M. Tartakovsky, S. P. Neuman, Extension of transient flow in bounded randomly heterogeneous domains. 1. Exact conditional moment equations and recursive approximations, Water Resour. Res., 35(6) (1999) 1921-1925.

[16] A. G. Madera, Modelling of stochastic heat transfer in a solid, Appl. Math. Model. 17 (1993) 664-668.

[17] A. G. Madera, A. N. Sotnikov, Method for analyzing stochastic heat transfer in a fluid flow, Appl. Math. Model. 20 (1996) 588-592.

[18] B. Oksendal, Stochastic Differential Equations, Springer, Berlin, 1998.

[19] T.T. Soong, Random Differential Equations in Science and Engineering, Academic Press, New York, 1973.

[20] E. Morales-Casique, S. P. Neuman, A. Guadagnini, Nonlocal and localized analyses of nonreactive solute transport in bounded randomly heterogeneous porous media: Theoretical framework, Adv. Water Res., 29 (2006) $1238-1255$. 
[21] E. Morales-Casique, S. P. Neuman, A. Guadagnini, Nonlocal and localized analyses of nonreactive solute transport in bounded randomly heterogeneous porous media: Computational analysis, Adv. Water Res., 29 (2006) 1399-1418.

[22] E. Morales-Casique, S.P. Neuman, Laplace-Transform Finite Element Solution of Nonlocal and Localized Stochastic Moment Equations of Transport, Commun. Comput. Phys., 6(1) (2009) 131-161.

[23] M.-C. Casabán, J.-C. Cortés, B. García-Mora, L. Jódar, AnalyticNumerical Solution of Random Boundary Value Heat Problems in a SemiInfinite Bar, Abstr. Appl. Anal., vol. 2013, Article ID 676372, 9 pages, 2013. doi:10.1155/2013/676372.

[24] M.-C. Casabán, R. Company, J.-C. Cortés, L. Jódar, Solving the random diffusion model in an infinite medium: A mean square approach, Appl. Math. Model. 38 (2014) 5922-5933.

[25] S. Peng, A stochastic Laplace transform for adapted processes and related BSDEs, Optimal Control and Partial Differential Equations, IOS Press (2001) 283-292.

[26] L. Na, W. Zhen, Stochastic transforms for jump diffusion processes combined with related BSDEs, J. Math. Anal. Appl. (2015), http://dx.doi.org/10.1016/j.jmaa.2014.12.033 (in press).

[27] L.T. Santos, F.A. Dorini, M.C.C. Cunha, The probability density function to the random linear transport equation, Appl. Math. Comput. 216 (2010) $1524-1530$.

[28] L. Villafuerte, C.A. Braumann, J.-C. Cortés, L. Jódar, Random differential operational calculus: theory and applications, Comput. Math. Appl. 59 (2010) 115-125.

[29] L. Arnold, Stochastic Differential Equations Theory and Applications, John Wiley, New York, 1974.

[30] J.-C. Cortés, L. Jódar, R. Company, L. Villafuerte, Solving Riccati timedependent models with random quadratic coefficients, Appl. Math. Lett. 24 (2011) 2193-2196.

[31] E.C. Titchmarsch, The Theory of Functions, 2th ed., Oxford University Press, New York, 1993.

[32] P. Henrici, Applied and Computational Complex Analysis. Volume I. John Wiley \& Sons, New York, 1974.

[33] M. Loève, Probability theory I and II, Graduate Tests in Mathematics, 4th ed., vol. 45, Springer-Verlag, 1977. 
423

[34] S. Sacks, A. Zygmund, Analytic Functions, third edition, Elsevier, Amsterdam, 1971.

[35] I.S. Gradshteyn, I.M. Ryzhik, Table of Integrals, Series, and Products, fifth edition, Academic Press, New York, 1994.

[36] G.B. Folland, Fourier Analysis and Its Applications, The Wadsworth \& Brooks/Cole Mathematics Series, Pacific Grove, California, 1992.

[37] G. Calbo, J.-C. Cortés, L. Jódar, Random Hermite differential equations: Mean square power series solutions and statistical properties, Appl. Math. Comput. 218 (2011) 3654-3666. 